\title{
The HaCaT/THP-1 Cocultured Activation Test (COCAT) for Skin Sensitization: A Study of Intra- Laboratory Reproducibility and Predictivity
}

\author{
Chantra Eskes ${ }^{1}$, Jennifer Hennen ${ }^{2}$, Mario T. Schellenberger2, Sebastian Hoffmann'3 , Sabine Frey 4 , \\ Daniela Goldinger-Oggier ${ }^{4}$, Niklas Peter ${ }^{2}$, Erwin van Vliet ${ }^{1}$ and Brunhilde Blömeke ${ }^{2}$ \\ ${ }^{1}$ Services \& Consultations on Alternative Methods, Magliaso, Switzerland; ${ }^{2}$ Department of Environmental Toxicology, Trier University, Trier, Germany; \\ $3^{3}$ seh consulting + services, Paderborn, Germany; ${ }^{4}$ Chemicals, Consumer Protection Directorate, Federal Office of Public Health (FOPH), Liebefeld, \\ Switzerland
}

\begin{abstract}
The Cocultured Activation Test (COCAT) consists of cocultured HaCaT (human keratinocyte cell line) and THP-1 cells (surrogate of antigen presenting cells). Individually, these cell lines are used to address key events 2 and 3 of the skin sensitization adverse outcome pathway (AOP). Their exposure in coculture was found to have the potential to increase their response to sensitizing chemicals, enable the detection of pro-haptens, and support the identification of skin sensitization potency. The present study was undertaken to assess the predictive capacity of COCAT of both skin sensitization hazard and potency and to assess the intra-laboratory reproducibility of COCAT based on the blind testing of chemicals. Results showed a reproducibility between runs of $80 \%$ for 15 coded chemicals. Skin sensitization hazard prediction had $100 \%$ sensitivity (9/9), 75\% specificity (3/4), and $92.3 \%$ accuracy (12/13), while the tests of two chemicals were inconclusive. Including additional chemicals tested during the optimization phase in addition to the blind tested chemicals, the skin sensitization UN GHS sub-categories were correctly predicted for $85.7 \%(12 / 14)$ sub-category 1 A chemicals, $83.3 \%(10 / 12)$ sub-category $1 \mathrm{~B}$ chemicals, and $92.3 \%(12 / 13)$ no category chemicals, resulting in an overall accuracy of $87.4 \%$ (34/39). The present study shows the COCAT to be a promising method for the identification of skin sensitization hazard and potency sub-categorization according to the UN GHS classification.
\end{abstract}

\section{Introduction}

Skin sensitization induced by chemicals represents an important endpoint for consumer and occupational safety assessment. For regulatory purposes, it can be characterized by hazard categorization according to, e.g., the UN GHS classification scheme (UN, 2017) and/or by quantitative risk assessment, for which a deeper understanding of the potency of the sensitizer is needed (as, e.g., for cosmetic ingredients). Traditionally, experimental animal models have been used to assess the skin sensitizing properties of chemicals (Api et al., 2015; Basketter et al., 2003). However, the demand for non-animal methods to determine skin sensitization hazard and potency is becoming increasingly urgent in order to comply with current regulations such as the Cosmetics Regulation 1223/2009 (EU, 2009), which prohibits animal testing for finished cosmetic products (since 2004) and ingredients (since 2009). In addition, an update to the REACH
Regulation requires that skin sensitization hazard and potency of chemicals is assessed by a combination of in vitro and in chemico studies and that in vivo testing is conducted only as a last resort (EU, 2016).

Given the complexity of the biological mechanisms underlying skin sensitization, it is generally recognized that a combination of mechanistically-based test methods is needed for both skin sensitization hazard assessment and potency prediction (Jowsey et al., 2006; OECD, 2014). Considerable progress has been made in the last decade regarding the development, validation, and adoption of alternative methods for skin sensitization hazard identification. In particular, a number of OECD Test Guidelines (TG) have been adopted that address the three major key events of the adverse outcome pathway (AOP) leading to skin sensitization. These are i) the covalent binding of electrophilic substances to nucleophilic centers in skin proteins as the molecular initiating event or first key event (OECD, 2019),
Received May 3, 2019; Accepted May 17, 2019

Epub May 27, 2019; (C) The Authors, 2019.

ALTEX 36(4), 613-622. doi:10.14573/altex.1905031

Correspondence: Brunhilde Blömeke, PhD,

Department of Environmental Toxicology, Trier University,

Universitätsring 15, 54296 Trier, Germany

(bloemeke@uni-trier.de)
This is an Open Access article distributed under the terms of the Creative Commons Attribution 4.0 International license (http://creativecommons.org/licenses/by/4.0/), which permits unrestricted use, distribution and reproduction in any medium, provided the original work is appropriately cited. 
ii) keratinocyte activation as the second key event (OECD, 2018b), and iii) activation of dendritic cells as the third key event (OECD, 2018a). In addition, OECD Guidance Documents 255 and 256 (OECD, 2017a,b) have been adopted, which describe the use of integrated approaches for testing and assessment (IATA) and of defined approaches (DA) to assess skin sensitization hazard or potency of chemicals. To date, however, no alternative test method has been adopted to sub-categorize skin sensitizers into subcategories 1A and 1B as defined by the UN GHS (UN, 2017) or for determining skin sensitization potency.

Trier University, in collaboration with the Swiss Federal Office of Public Health, has developed a coculture model composed of HaCaT keratinocytes and THP-1 dendritic cell-like cells, namely the HaCaT/THP-1 Cocultured Activation Test (COCAT), which was found promising to identify skin sensitizers (Hennen et al., 2011; Hennen and Blömeke, 2017a). Furthermore, the in vitro COCAT method was also shown to be able to identify the potency of skin sensitizers (Hennen and Blömeke, 2017a) and rank structurally related hair dye molecules (Hennen and Blömeke, 2017b, 2018). Further, coculture of THP-1 cells with reconstructed human epidermis (RHE) takes penetration of the compounds into consideration (Schellenberger et al., 2019; Hennen et al., 2019).

The COCAT combines two cell types that are individually used in two OECD TGs that address key events 2 and 3 of the skin sensitization AOP (i.e., $\mathrm{HaCaT}$ as main component of the OECD TG 442D for the KeratinoSens ${ }^{\mathrm{TM}}$ assay, and THP-1 as main component of the h-CLAT assay of the OECD TG 442E). This coculture allows a cross-talk between the two cell types and has been found, from preliminary data, to have the potential to:

- enhance the response of THP-1 cells to sensitizing agents, leading to higher sensitivity;

- detect pro-haptens;

- support the identification of skin sensitization potency as shown by correlation with Local Lymph Node Assay (LLNA) potency.

As information on skin sensitization potency is key in risk assessment of consumer and occupational exposures, a study was conducted to obtain data on the capacity of the COCAT to predict both skin sensitization hazard and potency, using an optimized test protocol. In this study, blind testing of chemicals was conducted in order to evaluate the intra-laboratory (between-run) reproducibility of the COCAT method and to assess its predictive capacity. The results obtained in the blind study, assessed both independently and combined with earlier results from the optimization phase, are presented here.

\section{Material and methods}

\section{Test chemicals}

The test chemicals oxazolone, citral, 2,4-dinitrochlorobenzene, 3-aminophenol, cinnamic aldehyde, tetramethylthiuram disulfide, 2-methoxy-4-methylphenol, eugenol, geraniol, cinnamic alcohol, vanillin, lactic acid, N,N-diethyl-3-methylbenzamide, 4-nitrobenzylbromide, 1-naphthol, R-carvone, toluene-2,5-diamine (sulfate), and limonene were obtained from Sigma Aldrich (Taufkirchen, Germany). N'-bis(4-aminophenyl)-2,5-diamino-1,4-quinonediimine (Bandrowski's base) was purchased from ICN Biomedicals (Aurora, OH, USA). Sodium dodecyl sulfate and dimethyl sulfoxide were purchased from Carl Roth (Karlsruhe, Germany). 4-Amino-2-methylacetanilide, 4-amino-3-methylacetanilide, 2,5diacetaminotoluene and 2-methoxymethyl- $p$-phenylenediamine, $\mathrm{N}$-[4-amino-3-(methoxymethyl)phenyl]acetamide, N,N'-(2-(methoxymethyl)-1,4-phenylene)diacetamide were kindly provided by Procter and Gamble (P\&G, Darmstadt, Germany). The chemicals tested in the blind study (diphenylcyclopropenone, $p$-phenylenediamine, formaldehyde, methyldibromo glutaronitrile, isoeugenol, 2-mercaptobenzothiazole, resorcinol (benzene-1,3-diol), coumarin, linalool, p-aminobenzoic acid, benzalkonium chloride, glycerol, salicylic acid, hydrocortisone, and propylparaben) were provided by VITO (Mol, Belgium).

\section{The COCAT}

The optimized Standard Operating Procedure (SOP version 9.5, Supplementary file ${ }^{1}$ ) of the COCAT was used. Briefly, HaCaT cells were cultured in DMEM supplemented with $10 \%$ FCS and $1 \%$ antibiotics solution (complete HaCaT culture medium). THP-1 cells were cultured in RPMI supplemented with $10 \% \mathrm{FCS}, 25 \mathrm{mM}$ HEPES, $4 \mathrm{mM}$ L-glutamine, $50 \mu \mathrm{M} \beta$-mercaptoethanol, and 1\% antibiotics solution (complete THP-1 culture medium). On day $1, \mathrm{HaCaT}$ cells were harvested, counted, and seeded in 96-well plates as $2.5 \times 10^{4}$ cells in $200 \mu 1$ complete $\mathrm{HaCaT}$ culture medium per well. Cells were cultivated for $48 \mathrm{~h}$ at which time they were $100 \%$ confluent. On day 3, THP-1 cells were harvested, counted, and added to the wells containing confluent $\mathrm{HaCaT}$ cells $\left(8 \times 10^{4}\right.$ THP- 1 cells in a total volume of $180 \mu 1$ exposure medium (complete THP-1 culture medium excluding $\beta$-mercaptoethanol) per well, incl. test chemicals). Test chemicals were freshly dissolved, diluted (serial 2-fold dilution, 7.8-4000 $\mu \mathrm{M}$ ), and added to the cells. $20 \mu \mathrm{M}$ 2,4-dinitrochlorobenzene (DNCB) and $144 \mu \mathrm{M}$ sodium dodecyl sulfate (SDS) was used as positive and negative control, respectively. Solvents used for dilution were dimethyl sulfoxide (DMSO, final concentration of $0.2 \%$ ) or cell culture medium. The maximal test concentration (up to $4000 \mu \mathrm{M}$ ) for each test chemical was determined based on its solubility.

After $24 \mathrm{~h}$ treatment, floating THP-1 cells were harvested, washed with phosphate buffered saline (PBS), subdivided into 2 subsamples for each well, and stained with FITC- and APC-labelled anti-CD86 (clone 2331 [FUN-1]) and anti-CD54 antibodies (clone HA58), respectively, or corresponding isotype controls (all mouse IgG1, obtained from BD Pharmingen, Heidelberg, Germany). Cell surface expression of CD86 and CD54 on viable THP-1 cells was analyzed by flow cytometry using a FACSVerse ${ }^{\mathrm{TM}}$ (BD, Heidelberg, Germany) followed by analysis with BD FACSuite ${ }^{\mathrm{TM}}$ (BD Biosciences, Heidelberg, Germany). Viability of THP-1 cells was determined by exclusion of propidium iodide (PI, $10 \mu \mathrm{g} / \mathrm{ml}$ ). For each sample, the mean fluores-

1 doi:10.14573/altex.1905031s 


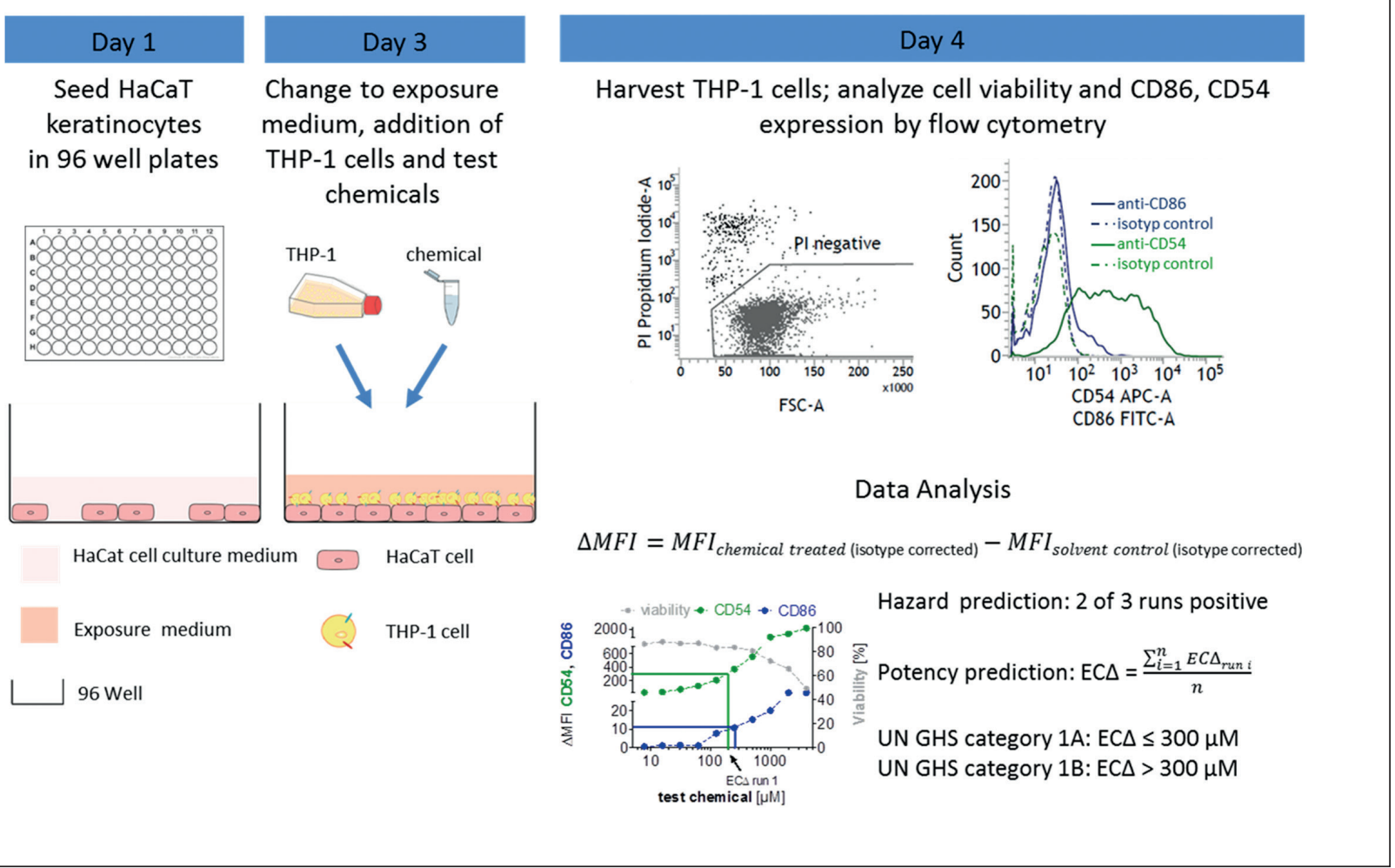

Fig. 1: Schematic view of the workflow of the COCAT test procedure

cence intensity (MFI) of the isotype control stained subsample was subtracted from the MFI of the corresponding anti-CD86 or anti-CD54 stained subsample. Then, $\triangle$ MFI reflecting the absolute difference between chemical-treated cells and solvent-treated cells was calculated by subtraction. A chemical is considered a sensitizer if in $\geq 2$ out of 3 runs at least one marker reaches its threshold for positivity $(\triangle \mathrm{MFI} \geq 10.8$ for $\mathrm{CD} 86$ or $\triangle \mathrm{MFI} \geq$ 300 for CD54) at cell viability $>50 \%$. A negative result obtained with a chemical that cannot be tested up to $4000 \mu \mathrm{M}$ due to solubility issues was considered "inconclusive".

For estimation of sensitizing potency, the lowest concentration reaching positivity for CD54 or CD86 in each individual run (total of three runs) was calculated by linear interpolation or log-linear extrapolation in the case that the $\Delta \mathrm{MFI}$ value at the lowest tested concentration already exceeded the threshold for positivity. The mean of these values is designated as the effective concentration (EC $\Delta$, Fig. 1).

\section{Design of the blind study}

Following protocol optimization, an intra-laboratory blind study was conducted to evaluate the reproducibility between the runs of an experiment and the preliminary predictive capacity of the COCAT. The primary goal of the study was to evaluate the ability of the COCAT to reliably support the discrimination of skin sensitizers from non-sensitizers, and furthermore to subcategorize skin sensitizers according to the UN GHS classification system.
15 chemicals for which reliable reference LLNA data were available were selected and tested in the blind study. For each test chemical, one experiment comprising three independent runs (conducted on different days) and triplicates within each run was conducted.

Testing was conducted in accordance with OECD GLP principles as much as possible, such as, but not limited to, use of SOP, compliant equipment and materials, adequate data recording, and record keeping.

\section{Chemical selection}

The chemical selection was conducted independently by Services \& Consultations on Alternative Methods (SeCAM, Switzerland), the Chemicals, Consumer Protection Directorate, Federal Office of Public Health (Switzerland), and seh consulting + services (Germany), safeguarding that the test developer and study laboratory (Trier University) was blinded to the test chemicals' identities. Table 1 shows the list of the 15 chemicals, which were selected using the following criteria:

- High quality reference LLNA and human data;

- Balanced distribution between sensitizers and non-sensitizers;

- Representation of various skin sensitization potencies;

- Inclusion of both haptens and pro-haptens;

- Wide range of chemistry, use/function and physico-chemical properties; 
Tab. 1: Predictive capacity of COCAT for the 15 blinded test chemicals

\begin{tabular}{|c|c|c|c|c|c|c|c|}
\hline \multirow[t]{2}{*}{ Test chemical } & \multirow[t]{2}{*}{ CAS \# } & \multirow{2}{*}{$\begin{array}{l}\text { Pro-/pre- } \\
\text { hapten }\end{array}$} & \multirow{2}{*}{$\begin{array}{l}\text { LLNA cat. } \\
(\text { EC3 in \%)* }\end{array}$} & \multirow{2}{*}{$\begin{array}{l}\text { Human skin } \\
\text { sensitization } \\
\text { potency } \\
\text { category ** }\end{array}$} & \multicolumn{2}{|c|}{ Positive runs } & \multirow[t]{2}{*}{ COCAT prediction } \\
\hline & & & & & \begin{tabular}{|l|} 
CD86 \\
\end{tabular} & CD54 & \\
\hline Diphenylcyclopropenone & $886-38-4$ & Hapten & Extreme $(0.05)$ & 1 & 3 of 3 & 3 of 3 & Sensitizer \\
\hline$p$-Phenylenediamine & $106-50-3$ & Prehapten & Extreme $(0.07)$ & 1 & 3 of 3 & 3 of 3 & Sensitizer \\
\hline Formaldehyde & $50-00-0$ & Hapten & Strong (0.4) & 2 & 3 of 3 & 3 of 3 & Sensitizer \\
\hline Methyldibromo glutaronitrile & $35691-65-7$ & Hapten & Strong (0.9) & 2 & 3 of 3 & 3 of 3 & Sensitizer \\
\hline Isoeugenol & $97-54-1$ & Prehapten & Moderate (1.2) & 2 & 3 of 3 & 3 of 3 & Sensitizer \\
\hline 2-Mercaptobenzothiazole & $149-30-4$ & Hapten & Moderate (1.7) & 3 & 0 of 3 & 3 of 3 & Sensitizer \\
\hline Coumarin & $91-64-5$ & Prohapten & Weak (30) & 3 & 3 of 3 & 0 of 3 & Sensitizer \\
\hline Resorcinol & $108-46-3$ & Prohapten & Moderate (6.3) & 4 & 2 of 3 & 3 of 3 & Sensitizer \\
\hline Linalool & $78-70-6$ & Prehapten & Weak (30) & 4 & 0 of 3 & 2 of 3 & Sensitizer \\
\hline p-Aminobenzoic acid & $150-13-0$ & - & Non-sensitizer & 5 & 0 of 3 & 1 of 3 & Non-sensitizer \\
\hline Benzalkonium chloride & $8001-54-5$ & - & Non-sensitizer & 5 & 0 of 3 & 1 of 3 & Non-sensitizer \\
\hline Hydrocortisone & $50-23-7$ & - & Non-sensitizer & 5 & 0 of 3 & 0 of 3 & Inconclusive \\
\hline Propylparaben & 94-13-3 & - & Non-sensitizer & 5 & 0 of 3 & 0 of 3 & Inconclusive ${ }^{* * *}$ \\
\hline Glycerol & $56-81-5$ & - & Non-sensitizer & 6 & 0 of 3 & 0 of 3 & Non-sensitizer \\
\hline Salicylic acid & 69-72-7 & - & Non-sensitizer & 6 & 3 of 3 & 0 of 3 & Sensitizer \\
\hline
\end{tabular}

${ }^{*}$ According to Urbisch et al. (2015); ${ }^{\star \star}$ According to Basketter et al. (2014); ${ }^{\star \star \star}$ Negative in COCAT up to the limit of solubility

- Exclusion of overly dangerous test chemicals, such as explosive chemicals;

- Exclusion of unstable chemicals, such as oxidizing and polymerizing agents;

- Exclusion of overly hazardous test chemicals, such as carcinogens, reproductive toxicants, and mutagens.

Acquisition, coding and distribution of chemicals was conducted independently by VITO (Belgium) using appropriate packaging. Information received by Trier University on the chemicals to be tested comprised the approximate molecular weight (MW), the physical form, and storage conditions. In addition, sealed envelopes containing health and safety information were dispatched to the Safety Officer appointed by Trier University (Dr Udo Bock). The sealed envelopes were sent back to SeCAM after the experimental study phase, where it was confirmed that no envelopes had been opened.

Data collection, handling, and analyses

The data collection spreadsheets used for reporting the study data were prepared by Trier University and reviewed by the biostatistician of the study. The raw data produced during the blind study by Trier University were provided to the study biostatistician, who collected, managed, and analyzed the data. After hazard prediction and unblinding of the coded test chemicals, the data were reanalyzed with the corrected MW.

Data processed according to the SOP to obtain mean cell viability, mean CD86 $\triangle \mathrm{MFI}$, and mean CD54 $\triangle \mathrm{MFI}$ for each test concentration, run, and chemical were analyzed. Reproducibility of runs of an experiment (composed of three valid runs conducted on different days) was analyzed descriptively comparing solubility and cytotoxicity of tested concentrations, and lowest positive concentration for both $\triangle \mathrm{MFI}(\mathrm{CD} 86 \geq 10.8$; CD54 $\geq 300)$. In addition, concordance of classification of runs (non-sensitizer $v s$ sensitizer) as obtained with the prediction model was calculated for the 15 test chemicals.

\section{Results}

\subsection{Predictive capacity}

Each test chemical was assessed in the COCAT in three individual runs. The concentration-dependent upregulation of CD86 and CD54, and the reduction of cell viability of THP-1 cells in each run are shown in Figure 1 for three exemplary chemicals.

The predictive capacity of the COCAT in the blind study was assessed by comparing the in vitro predictions with LLNA and human reference results, from which identical skin sensitization potential/hazard was concluded for the 15 tested chemicals. As shown in Table 1, the nine skin sensitizers were all correctly predicted in the COCAT, mainly driven by CD54. Also, three of the six non-sensitizers were correctly predicted. Salicylic acid was identified as a false positive in the COCAT, whereas no conclusion could be drawn for the two remaining non-sensitizers (hydrocortisone and propylparaben) due to limited solubility (incon- 


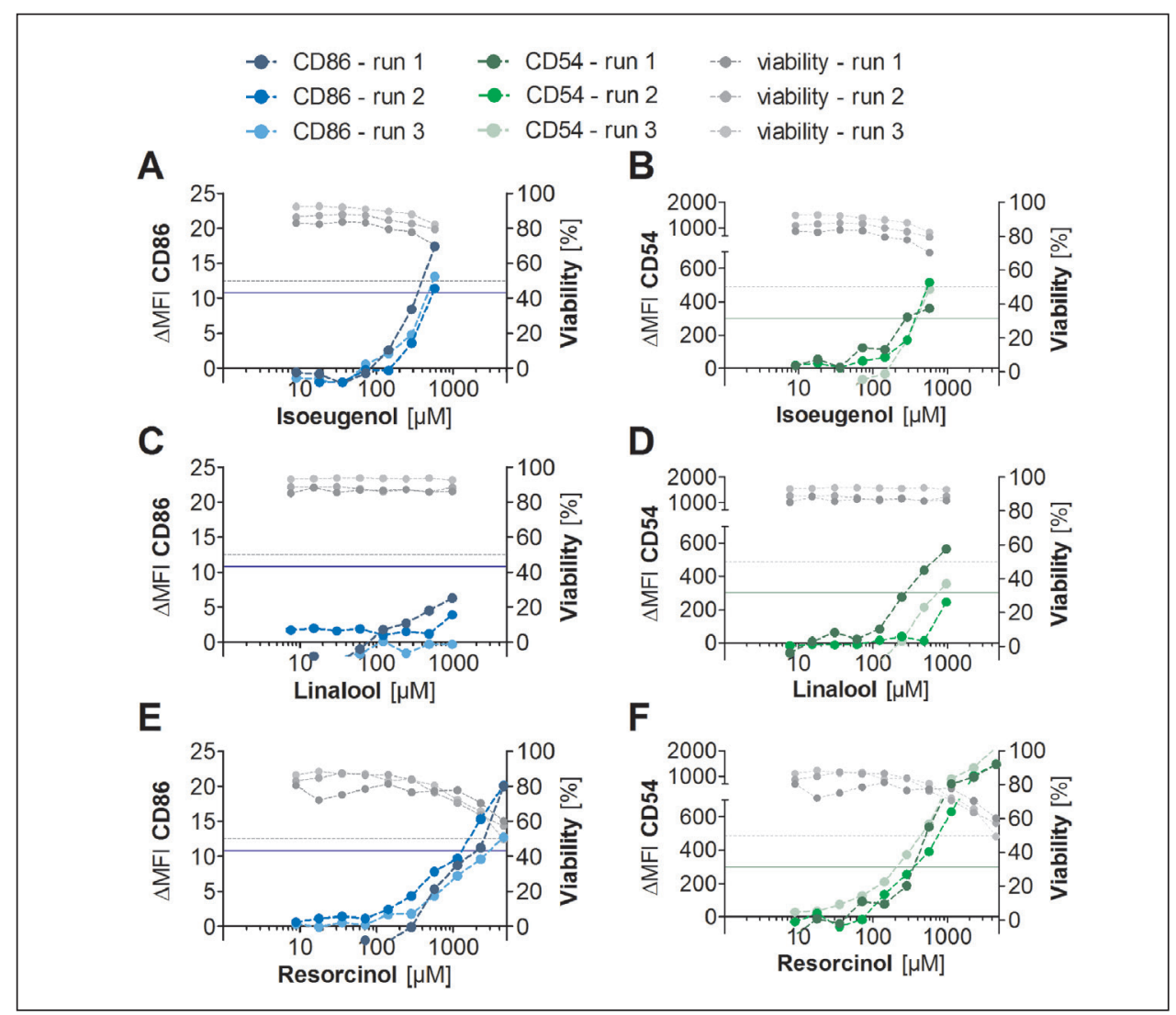

Fig. 2: Concentrationdependent up-regulation of CD86 (A, C, and E) and CD54 (B, D, and F) on viable THP-1 cells in the COCAT after $24 \mathrm{~h}$ chemical exposure

The individual runs for isoeugenol $(A, B)$, linalool $(C, D)$, and resorcinol $(E, F)$ as representative chemicals are shown. The dashed line represents the limit of viability accepted in the COCAT (i.e., reduction of viability to $50 \%$ ), the blue and green horizontal lines represent the threshold for positivity for CD86 or CD54 with a $\triangle \mathrm{MFI}$ of 10.8 or 300 , respectively.

clusive). In summary, the COCAT correctly predicted 12 out of 13 (excluding hydrocortisone and propylparaben due to reduced solubility) test chemicals, i.e., $92 \%$ when compared to the LLNA reference data. When compared to the human data, the same predictive capacity was observed if human categories 1 to 4 are considered sensitizers and human categories 5 and 6 are considered non-sensitizers as described by Basketter et al. (2014).

\subsection{Reproducibility}

Reproducibility between runs of an experiment (composed of at least three valid runs conducted on different days and containing triplicate measurements within each run) was analyzed descriptively by comparing cytotoxicity of test concentrations and lowest positive concentration for both $\triangle \mathrm{MFI}$ (CD86 $\geq 10.8$; CD54 $\geq 300$, Tab. S $\left.{ }^{1}\right)$. In addition, concordance of classification of runs (non-sensitizer $v s$ sensitizer) as obtained with the prediction model was calculated across the 15 test chemicals.

As shown in Table $\mathrm{S}^{1}{ }^{1}$, the cytotoxicity between runs was identical for 12 of the 15 test chemicals (i.e., 80\%) when using the lowest concentration leading to a compound-induced reduction of cell viability of not more than $35 \%$ as determined by propidium iodide staining. Minor differences were observed for methyldibromo glutaronitrile (difference by a factor of 2 , i.e., one dilution step) and $p$-phenylenediamine (difference by a factor of 4). Resorcinol gave a cell viability of $49.3 \%$ (just below the cut-off value of $50 \%$ ) for the highest test concentration in the third run.
The induction of CD86 and CD54 was well reproducible between runs for most chemicals when considering the lowest concentrations inducing these markers above the respective thresholds (Fig. 2, Tab. S11). Regarding CD86, clear differences were observed for two chemicals ( $p$-phenylenediamine and coumarin). The CD86 reproducibility of resorcinol was affected by the differences in cytotoxicity between the runs (the borderline cytotoxic concentration of $4540.9 \mu \mathrm{M}$ induced CD86 above the threshold). Regarding CD54, there were clear differences between the runs for $p$-phenylenediamine and linalool, while the positive run for benzalkonium chloride was only borderline positive.

In summary, the reproducibility of runs when testing 15 coded chemicals was $80 \%$ or higher for cytotoxicity and for the two cell surface markers, regardless of the analysis (concentration and prediction).

\subsection{Prediction of UN GHS sub-categories 1 A and 1 B}

Beside hazard identification, the capacity of the COCAT to categorize chemicals according to their skin sensitizing potency was also assessed in this study. To enlarge the database for the assessment of the capacity of COCAT to discriminate UN GHS skin sensitization sub-categories $1 \mathrm{~A}$ vs $1 \mathrm{~B}$, results of the blind study were combined with results obtained earlier (published in Goebel et al., 2014; Hennen and Blömeke, 2017a,b, 2018; unpublished data), yielding a total of 26 skin sensitizers and 13 non-sensitizers. These earlier results were obtained using a 6-well format protocol or a protocol with minor variations 
Tab. 2: Overview of the compiled results for hazard, UN GHS sub-categories and potency prediction

\begin{tabular}{|c|c|c|c|c|c|}
\hline \multirow[t]{2}{*}{ Test chemical } & \multirow[t]{2}{*}{ CAS \# } & \multirow{2}{*}{$\begin{array}{l}\text { UN GHS } \\
\text { cat.* }^{*}\end{array}$} & \multirow[t]{2}{*}{$\mathrm{EC} \Delta[\mu \mathrm{M}]$} & \multicolumn{2}{|c|}{ COCAT prediction } \\
\hline & & & & $\begin{array}{l}\text { Predicted } \\
\text { hazard }\end{array}$ & $\begin{array}{l}\text { Predicted UN } \\
\text { GHS cat. }\end{array}$ \\
\hline \multicolumn{6}{|l|}{ Sensitizers } \\
\hline Oxazolone & $15646-46-5$ & $1 \mathrm{~A}$ & 73.8 & Sensitizer & $1 \mathrm{~A}$ \\
\hline Bandrowski's base & $20048-27-5$ & $1 \mathrm{~A}$ & $6.7^{\mathrm{b}}$ & Sensitizer & $1 \mathrm{~A}$ \\
\hline 2,4-Dinitrochlorobenzene & $97-00-7$ & $1 \mathrm{~A}$ & 13.9 & Sensitizer & $1 \mathrm{~A}$ \\
\hline 4-Nitrobenzyl bromide & $100-11-8$ & $1 \mathrm{~A}$ & 5.9 & Sensitizer & $1 \mathrm{~A}$ \\
\hline Diphenylcyclopropenone (blind study) & $886-38-4$ & $1 \mathrm{~A}$ & 6.9 & Sensitizer & $1 \mathrm{~A}$ \\
\hline p-Phenylenediamine (blind study) & $106-50-3$ & $1 \mathrm{~A}$ & 71.8 & Sensitizer & $1 \mathrm{~A}$ \\
\hline Toluene-2,5-diamine & $615-50-9$ & $1 \mathrm{~A}$ & $193.2^{b}$ & Sensitizer & $1 \mathrm{~A}$ \\
\hline Formaldehyde (blind study) & $50-00-0$ & $1 \mathrm{~A}$ & 87.2 & Sensitizer & $1 \mathrm{~A}$ \\
\hline Methyldibromo glutaronitrile (blind study) & $35691-65-7$ & $1 \mathrm{~A}$ & 16.3 & Sensitizer & $1 \mathrm{~A}$ \\
\hline Isoeugenol (blind study) & $97-54-1$ & $1 \mathrm{~A}$ & 362.7 & Sensitizer & 1B \\
\hline 1-Naphthol & $90-15-3$ & $1 \mathrm{~A}$ & $189.0^{\mathrm{C}}$ & Sensitizer & $1 \mathrm{~A}$ \\
\hline 2-Mercaptobenzothiazole (blind study) & $149-30-4$ & $1 \mathrm{~A}$ & 97.1 & Sensitizer & $1 \mathrm{~A}$ \\
\hline 3-Aminophenol & $591-27-5$ & 1B & 566.1 & Sensitizer & 1B \\
\hline Cinnamic aldehyde & $104-55-2$ & $1 \mathrm{~A}$ & $140.8^{\mathrm{a}}$ & Sensitizer & $1 \mathrm{~A}$ \\
\hline 2-Methoxymethyl-para-phenylenediamine & $337906-36-2$ & 1B & 1812.1 & Sensitizer & 1B \\
\hline Citral & $5392-40-5$ & $1 \mathrm{~B}$ & 84.7 & Sensitizer & $1 \mathrm{~A}$ \\
\hline Tetramethylthiuram disulfide & $137-26-8$ & $1 \mathrm{~B}$ & 30.5 & Sensitizer & $1 \mathrm{~A}$ \\
\hline 2-Methoxy-4-methylphenol & $93-51-6$ & $1 \mathrm{~B}$ & 504.9 & Sensitizer & $1 \mathrm{~B}$ \\
\hline Resorcinol (blind study) & $108-46-3$ & 1B & 325.0 & Sensitizer & 1B \\
\hline Eugenol & $97-53-0$ & $1 \mathrm{~B}$ & 427.2 & Sensitizer & $1 \mathrm{~B}$ \\
\hline R-Carvone & $6485-40-1$ & $1 \mathrm{~B}$ & 502.2 & Sensitizer & 1B \\
\hline Geraniol & $106-24-1$ & $1 \mathrm{~B}$ & 697.5 & Sensitizer & $1 \mathrm{~B}$ \\
\hline Cinnamic alcohol & $104-54-1$ & $1 \mathrm{~B}$ & 820.8 & Sensitizer & 1B \\
\hline Coumarin (blind study) & $91-64-5$ & $1 \mathrm{~B}$ & 963.2 & Sensitizer & $1 \mathrm{~B}$ \\
\hline Linalool (blind study) & $78-70-6$ & $1 \mathrm{~B}$ & 529.9 & Sensitizer & $1 \mathrm{~B}$ \\
\hline Limonene & $5989-27-5$ & 1B & 824.1 & Sensitizer & 1B \\
\hline \multicolumn{6}{|l|}{ Non-sensitizers } \\
\hline p-Aminobenzoic acid (blind study) & $150-13-0$ & No Cat. & - & Non-sensitizer & No Cat. \\
\hline Benzalkonium chloride (blind study) & $8001-54-5$ & No Cat. & - & Non-sensitizer & No Cat. \\
\hline Glycerol (blind study) & $56-81-5$ & No Cat. & - & Non-sensitizer & No Cat. \\
\hline Salicylic acid (blind study) & $69-72-7$ & No Cat. & 1276.0 & Sensitizer & $1 \mathrm{~B}$ \\
\hline N,N-Diethyl-3-methylbenzamid (DEET) & $134-62-3$ & No Cat. & $-{ }^{a}$ & Non-sensitizer & No Cat. \\
\hline Lactic acid & $50-21-5$ & No Cat. & $-{ }^{a}$ & Non-sensitizer & No Cat. \\
\hline Sodium dodecyl sulfate & $151-21-3$ & No Cat. & $-{ }^{a}$ & Non-sensitizer & No Cat. \\
\hline Vanillin & $121-33-5$ & No Cat. & $-^{a}$ & Non-sensitizer & No Cat. \\
\hline 4-Amino-2-methylacetanilide & $56891-59-9$ & No Cat. & $-^{a}$ & Non-sensitizer & No Cat. \\
\hline 4-Amino-3-methylacetanilide & $6375-20-8$ & No Cat. & $-{ }^{a}$ & Non-sensitizer & No Cat. \\
\hline 2,5-Diacetaminotoluene 1 & $19039-27-1$ & No Cat. & $-{ }^{a}$ & Non-sensitizer & No Cat. \\
\hline $\begin{array}{l}\mathrm{N}-[4-\text { Amino-3-(methoxymethyl)phenyl] } \\
\text { acetamide }\end{array}$ & n.a. & No Cat. & $-e^{d}$ & Non-sensitizer & No Cat. \\
\hline $\begin{array}{l}\text { N,N'-(2-(Methoxymethyl)-1,4-phenylene) } \\
\text { diacetamide }\end{array}$ & n.a. & No Cat. & $-e^{d}$ & Non-sensitizer & No Cat. \\
\hline
\end{tabular}

Shadowed cells relate to under- (orange) or over- (yellow) predictions. *based on LLNA EC3 values (Hoffmann et al., 2018; Urbisch et al., 2015; Rudback et al., 2014; Johansson et al., 2008; Goebel et al., 2014). ${ }^{a}$ EC $\Delta$ published in Hennen and Blömeke (2017a); ${ }^{b}$ EC $\Delta$ published in Hennen and Blömeke (2017b); ${ }^{c}$ EC $\Delta$ published in Hennen and Blömeke (2018); ' $\mathrm{EC} \Delta$ published in Goebel et al. (2014); n.a., not available. 


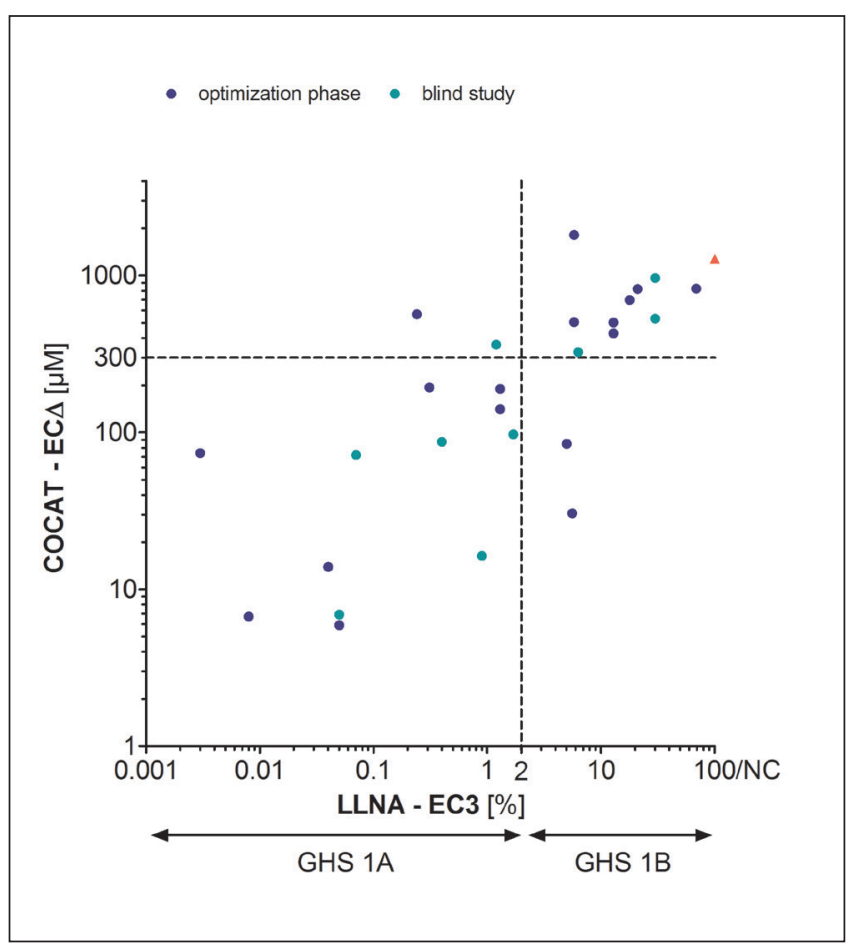

Fig. 3: Categorization of sensitizers into GHS sub-categories $1 \mathrm{~A}$ or $1 \mathrm{~B}$ according to EC $\Delta$ determined in the COCAT

Results for 26 sensitizers tested in the optimization phase (17 sensitizers, dark blue circle) or in the blind study ( 9 sensitizers, green circle and false positive tested chemical as red triangle) are shown. The calculated EC $\Delta$ are summarized in Table 3. NC, no category

in the selection of test concentrations in some cases, which were found to not impact on the overall result. The summary of all results for the 39 chemicals is shown in Table 2.

In order to assign sensitizers into UN GHS sub-categories $1 \mathrm{~A}$ or $1 \mathrm{~B}$ based on the lowest positive concentration in the $\mathrm{CO}$ CAT (ECA), a cut-off of $300 \mu \mathrm{M}$ was used (Fig. 3 and Tab. 3). Using this cut-off to distinguish skin sensitizers with UN GHS sub-category $1 \mathrm{~A}$ from $1 \mathrm{~B}$ allowed to correctly predict 12 of 13 skin sensitizers of sub-category $1 \mathrm{~A}$ and 11 of 13 skin sensitizers of sub-category 1B (comparing to sub-categorization based on LLNA EC3 values), representing a total of 23 of 26 correctly predicted sub-category $1 \mathrm{~A}$ or $1 \mathrm{~B}$ sensitizers $(85.5 \%)$. In addition, 12 of the 13 non-sensitizers were correctly predicted as such $(92.3 \%)$. One sub-category 1A sensitizer (out of 13) was under-predicted as sub-category 1B sensitizer. Furthermore, two sub-category 1B (out of 14) sensitizers were over-predicted as sub-category $1 \mathrm{~A}$ and one non-sensitizer was over-predicted as sub-category 1B (salicylic acid). No skin sensitizer yielded a false-negative result. Altogether, an overall concordance of $87.4 \%(34 / 39)$ correct predictions of UN GHS sub-categories (1A, 1B and non-sensitizers) was found, underlining the high capacity of COCAT for predicting potency sub-categories according to the UN GHS classification scheme.
Tab. 3: Contingency table of LLNA versus COCAT predictions

Discriminating between sub-category $1 \mathrm{~A}$, sub-category $1 \mathrm{~B}$, and no category using the cut-off of $300 \mu \mathrm{M}$ as the lowest concentration needed for positivity in COCAT (EC $\triangle$ ) to trigger prediction of sub-category $1 \mathrm{~A}$.

\begin{tabular}{|l|l|l|l|l|l|}
\cline { 3 - 6 } \multicolumn{2}{c|}{} & \multicolumn{2}{l}{ COCAT } & \multirow{2}{*}{ Total } \\
\cline { 2 - 5 } \multicolumn{2}{c|}{} & $1 \mathrm{~A}$ & $1 \mathrm{~B}$ & No Cat. & \\
\hline \multirow{3}{*}{ LLNA } & $1 \mathrm{~A}$ & 12 & 2 & 0 & 14 \\
\cline { 2 - 6 } & $1 \mathrm{~B}$ & 2 & 10 & 0 & 12 \\
\cline { 2 - 6 } & No Cat. & 0 & 1 & 12 & 13 \\
\hline \multirow{3}{*}{ Total } & 14 & 13 & 12 & 39 \\
\hline
\end{tabular}

\section{Discussion}

The identification and characterization of a chemical's potential to induce skin sensitization is a prerequisite for its risk assessment. The Cosmetics Regulation 1223/2009 (EU, 2009) prohibits animal testing for individual ingredients and finished products, and also the European Union's REACH Regulation (EU, 2016) demands the use of alternative methods where applicable. Furthermore, estimation of potency is critical for risk assessment and the sub-categorization of skin sensitizers according to their potency into UN GHS subcategories $1 \mathrm{~A}$ or $1 \mathrm{~B}$ is mandatory under the REACH Regulation (EU, 2016).

The regulatory accepted methods (OECD 2018a,b, 2019) have been validated for the purpose of hazard identification. Several defined approaches integrating results obtained from individual methods, each representing one key event of the AOP for skin sensitization, have been evaluated for their capacity to predict potency. However, there is considerable uncertainty about the application of the various combinations of non-animal methods for the assessment of chemical skin sensitization potential and potency for regulatory decision-making, e.g., for the purposes of REACH or classification according to the CLP Regulation (ECHA, 2018; EU, 2006, 2008, 2016).

In contrast to the regulatory accepted methods, the COCAT combines two cell types, i.e., HaCaT keratinocytes (included in OECD TG 442D addressing key event 2, activation of keratinocytes) and THP-1 cells (included in OECD TG 442E addressing key event 3, activation of dendritic cells) (Emter et al., 2010; Ramirez et al., 2014; Takahashi et al., 2011; Ashikaga et al., 2006). These two cell types are directly cocultured in the COCAT, allowing for co-exposure and cross-talk between them. Such interaction has been found to have the potential to enhance the response of THP-1 cells to sensitizing agents, and to improve the detection of pro-haptens, thus improving the sensitivity of the assay (Hennen and Blömeke, 2017a). Furthermore, the combination of dendritic cell activation with keratinocyte responses to skin sensitizing chemicals has been found to support the identification of skin sensitization potency (Hennen and Blömeke, 2017a, 2018; Goebel et al., 2014). Following 
Tab. 4: Comparison of COCAT hazard prediction with currently adopted monoculture assays that are individually used in two OECD test guidelines which address key events 2 (KeratinoSens ${ }^{\mathrm{TM}}$ ) and 3 (h-CLAT) of the skin sensitization AOP

\begin{tabular}{|c|c|c|c|c|}
\hline Test chemical & LLNA classification & $\begin{array}{l}\text { COCAT } \\
(\mathrm{HaCaT}+\mathrm{THP}-1)\end{array}$ & $\begin{array}{l}\text { TG 442D } \\
\text { KeratinoSens }{ }^{\mathrm{TM}} \\
\text { (HaCaT) }\end{array}$ & $\begin{array}{l}\text { TG 442E h-CLAT } \\
\text { (THP-1) }\end{array}$ \\
\hline Diphenylcyclopropenone & Extreme & Positive & Positive $^{1}$ & Positive $^{4}$ \\
\hline$p$-Phenylenediamine & Extreme & Positive & Positive $^{1}$ & Positive 4 \\
\hline Formaldehyde & Strong & Positive & Positive $^{1}$ & Positive $^{4}$ \\
\hline Methyldibromo glutaronitrile & Strong & Positive & Positive $^{1}$ & Positive $^{4}$ \\
\hline Isoeugenol & Moderate & Positive & Positive $^{1}$ & Negative $^{4}$ \\
\hline 2-Mercaptobenzothiazole & Moderate & Positive & Positive $^{1}$ & Positive $^{4}$ \\
\hline Resorcinol & Moderate & Positive & Negative $^{1}$ & Positive $^{4}$ \\
\hline Coumarin & Weak & Positive & Positive $^{1}$ & Negative $^{5}$ \\
\hline Linalool & Weak & Positive & Negative $^{1}$ & Positive $^{4}$ \\
\hline$p$-Aminobenzoic acid & Non-sensitizer & Negative & Negative $^{2}$ & Negative $^{3}$ \\
\hline Benzalkonium chloride & Non-sensitizer & Negative & Negative $^{1}$ & Negative $^{4}$ \\
\hline Glycerol & Non-sensitizer & Negative & Negative $^{1}$ & Negative $^{4}$ \\
\hline Salicylic acid & Non-sensitizer & Positive & Negative $^{1}$ & Positive $^{4}$ \\
\hline Correct predictions & & $12 / 13$ & $11 / 13$ & $10 / 13$ \\
\hline
\end{tabular}

${ }^{1}$ Natsch et al. (2013); ${ }^{2}$ Urbisch et al. (2015); ${ }^{3}$ Hoffmann et al. (2018); ${ }^{4}$ Nukada et al. (2012); ${ }^{5}$ Takenouchi et al. (2013)

this proof-of-principle, the test protocol was optimized to allow an increased throughput of the COCAT by using 96-well plates and to define the COCAT's critical protocol steps such as cell viability assessment, the prediction model used, the concentration ranges to be tested, definition of positive and negative controls, and the definition of a strategy for test chemical solubilization. The optimized protocol ${ }^{1}$ was then applied in this intra-laboratory pre-validation study. Results from the blind intra-laboratory phase of the present study, in which 15 coded chemicals were tested in three independent runs composed of triplicates within each run, showed the assay to be reproducible, achieving a reproducibility between runs of $80 \%$ (12 out of 15$)$ or higher for cytotoxicity and for the two markers (CD86 and CD54), regardless of the analysis (concentration and prediction). Furthermore, it showed the correct prediction of 9 out of 9 skin sensitizers, and of 3 out of 4 non-sensitizers, resulting in an overall accuracy of $92.3 \%$ (12/13) for LLNA and human hazard reference data. Comparison of the results of the blind study with predictions from currently adopted in vitro assays using HaCaT or THP-1 cells alone (i.e., KeratinoSens ${ }^{\mathrm{TM}}$, h-CLAT) demonstrated a similar or better performance of the COCAT (Tab. 4).

When combining the results obtained from the optimization and blind studies, a sensitivity of $100 \%(26 / 26)$, a specificity of $92.3 \%(12 / 13)$, and an overall accuracy of $97.4 \%(38 / 39)$ was achieved for the identification of skin sensitization hazards. Using $300 \mu \mathrm{M}$ as cut-off for distinguishing skin sensitizers of
UN GHS sub-category 1A from 1B allowed to correctly predict 23 out of the 26 sensitizers $(88.5 \%)$ and 12 of the 13 non-sensitizers $(92.3 \%)$, achieving an overall concordance of $87.4 \%$ (34/39) for predicting the UN GHS sub-categories 1A, 1B and non-sensitizers.

A detailed analysis of the data revealed that all but one chemical reached positivity for CD54 at a lower concentration than CD86. Consequently, potency prediction in COCAT was dominated by the chemicals' capacity to upregulate CD54. In line, the interaction of adhesion molecule CD54 and its counterpart (LFA-1) on T cells was found to not only mediate intercellular binding but also to deliver signals to T cells. Specifically, it was found to decrease the threshold of naïve $T$ cell activation and antigen dose required for T cell activation (Wang et al., 2008). This underlines the importance of the chemicals' potential to upregulate CD54 and its quantitative relationship with potency prediction, i.e., the amount of chemical required for the induction of skin sensitization. Nevertheless, CD54 is only addressed in h-CLAT and COCAT, while other assays such as U-SENS, the IL-8 Luc assay, or the GARD assay do not comprise the analysis of CD54 upregulation by chemicals (Wong et al., 2015; Ashikaga et al., 2006; Piroird et al., 2015; Takahashi et al., 2011; Johansson et al., 2013). However, the concentration needed for a sufficient upregulation of CD54 on THP-1 cells was found to be modulated by adjacent $\mathrm{HaCaT}$ keratinocytes by a factor of up to 9 in COCAT, crucially impacting on its capacity to estimate the 
chemicals' sensitizing potency (Hennen and Blömeke, 2017a). In the present study, comparison of the EC $\Delta$ values obtained in COCAT with LLNA EC3 values led to a highly comparable assignment of chemicals into UN GHS potency sub-categories (Tab. 3), and the results also indicate a potential of COCAT for a more refined potency prediction on a continuous scale (Fig. 3) as, e.g., needed for the quantitative risk assessment of chemicals such as cosmetic ingredients.

Overall, the present study shows the COCAT method, integrating two key events of the AOP, not only to be a reproducible method that is promising for the identification of skin sensitization hazard, but also its capacity for sub-categorization according to the UN GHS classification sub-categorization scheme and possibly potency in a more detailed manner. Thus, the COCAT has the potential to provide data needed to fulfil the updated information requirements of $\mathrm{REACH}$ and also to support quantitative risk assessment using non-animal methods for other regulatory purposes.

\section{References}

Api, A. M., Basketter, D. and Lalko, J. (2015). Correlation between experimental human and murine skin sensitization induction thresholds. Cutan Ocul Toxicol 34, 298-302. doi:10.31 09/15569527.2014.979425

Ashikaga, T., Yoshida, Y., Hirota, M. et al. (2006). Development of an in vitro skin sensitization test using human cell lines: The human Cell Line Activation Test (h-CLAT). I. Optimization of the h-CLAT protocol. Toxicol In Vitro 20, 767-773. doi:10.1016/j.tiv.2005.10.012

Basketter, D. A., Angelini, G., Ingber, A. et al. (2003). Nickel, chromium and cobalt in consumer products: Revisiting safe levels in the new millennium. Contact Dermatitis 49, 1-7. doi:10.1111/j.0105-1873.2003.00149.x

Basketter, D. A., Alepee, N., Ashikaga, T. et al. (2014). Categorization of chemicals according to their relative human skin sensitizing potency. Dermatitis 25, 11-21. doi:10.1097/ DER.0000000000000003

ECHA (2018). Background Document on in vitro testing for skin sensitisation. MSC-RAC Joint Workshop on Fine tuning the testing requirements and evaluation of selected human health endpoints under REACH and CLP. 11-12 October 2018, Helsinki. https://echa.europa.eu/de/about-us/who-we-are/memberstate-committee/meetings-of-the-member-state-committee/ other-meetings

Emter, R., Ellis, G. and Natsch, A. (2010). Performance of a novel keratinocyte-based reporter cell line to screen skin sensitizers in vitro. Toxicol Appl Pharmacol 245, 281-290. doi:10.1016/j.taap.2010.03.009

EU (2006). Regulation (EC) No 1907/2006 of the European Parliament and of the Council of 18 December 2006 concerning the Registration, Evaluation, Authorisation and Restriction of Chemicals (REACH), establishing a European Chemicals Agency, amending Directive 1999/45/EC and repealing Council Regulation (EEC) No 793/93 and Commission Regulation (EC) No 1488/94 as well as Council Directive 76/769/EEC and Commission Directives 91/155/EEC, 93/67/EEC, 93/105/ EC and 2000/21/EC. Off J Eur Union L396, 1-520. http://data. europa.eu/eli/reg/2006/1907/2014-04-10

EU (2008). Regulation (EC) No 1272/2008 of the European Parliament of the Council of 16 December 2008 on classification, labelling and packaging of substances and mixtures, amending and repealing Directives 67/548/EEC and 1999/45/EC, and amending Regulation (EC) No 1907/2006. Off J Eur Union L353, 1-1355. https://eur-lex.europa.eu/legal-content/EN/TXT /?uri=CELEX\%3A32008R1272

EU (2009). Regulation (EC) No. 1223/2009 of the European Parliament and of the Council of 30 November 2009 on cosmetic products. Off J Eur Union L342, 59-209. https://eur-lex. europa.eu/eli/reg/2009/1223/oj

EU (2016). Commission Regulation (EU) 2016/1688 of 20 September 2016 amending Annex VII to Regulation (EC) No 1907/2006 of the European Parliament and of the Council on the Registration, Evaluation, Authorisation and Restriction of Chemicals (REACH) as regards skin sensitisation. Off J Eur Union L255, 14-16. http://data.europa.eu/eli/reg/2016/1688/oj

Goebel, C., Troutman, J., Hennen, J. et al. (2014). Introduction of a methoxymethyl side chain into p-phenylenediamine attenuates its sensitizing potency and reduces the risk of allergy induction. Toxicol Appl Pharmacol 274, 480-487. doi:10.1016/j. taap.2013.11.016

Hennen, J., Aeby, P., Goebel, C. et al. (2011). Cross talk between keratinocytes and dendritic cells: Impact on the prediction of sensitization. Toxicol Sci 123, 501-510. doi:10.1093/toxsci/ kfr174

Hennen, J. and Blömeke, B. (2017a). Keratinocytes improve prediction of sensitization potential and potency of chemicals with THP-1 cells. ALTEX 34, 279-288. doi:10.14573/ altex. 1606171

Hennen, J. and Blömeke, B. (2017b). Assessment of skin sensitization potency of hair dye molecules in vitro. Contact Dermatitis 77, 179-180. doi:10.1111/cod.12780

Hennen, J. and Blömeke, B. (2018). Ranking skin-sensitizing hair dye molecules according to their potency by the use of human cells. Contact Dermatitis 79, 391-393. doi:10.1111/ cod. 13094

Hennen, J., Silva, E. S. M., Sahli, F. et al. (2019). Sensitization potential and potency of terpene hydroperoxides in the COCAT method. Contact Dermatitis 81, 97-103. doi:10.1111/cod.13286

Hoffmann, S., Kleinstreuer, N., Alepee, N. et al. (2018). Nonanimal methods to predict skin sensitization (I): The Cosmetics Europe database. Crit Rev Toxicol 48, 344-358. doi:10.108 0/10408444.2018.1429385

Johansson, H., Albrekt, A. S., Borrebaeck, C. A. et al. (2013). The GARD assay for assessment of chemical skin sensitizers. Toxicol In Vitro 27, 1163-1169. doi:10.1016/j.tiv.2012.05.019

Johansson, S., Gimenez-Arnau, E., Grotli, M. et al. (2008). Carbon- and oxygen-centered radicals are equally important haptens of allylic hydroperoxides in allergic contact dermatitis. Chem Res Toxicol 21, 1536-1547. doi:10.1021/tx800104c

Jowsey, I. R., Basketter, D. A., Westmoreland, C. et al. (2006). A future approach to measuring relative skin sensitising potency: 
A proposal. J Appl Toxicol 26, 341-350. doi:10.1002/jat.1146

Natsch, A., Ryan, C. A., Foertsch, L. et al. (2013). A dataset on 145 chemicals tested in alternative assays for skin sensitization undergoing prevalidation. J Appl Toxicol 33, 1337-1352. doi:10.1002/jat.2868

Nukada, Y., Ashikaga, T., Miyazawa, M. et al. (2012). Prediction of skin sensitization potency of chemicals by human Cell Line Activation Test (h-CLAT) and an attempt at classifying skin sensitization potency. Toxicol In Vitro 26, 1150-1160. doi:10.1016/j.tiv.2012.07.001

OECD (2014). The Adverse Outcome Pathway for Skin Sensitisation Initiated by Covalent Binding to Proteins. OECD Series on Testing and Assessment, No. 168. OECD Publishing, Paris. doi:10.1787/9789264221444-en

OECD (2019). Test No. 442C: In Chemico Skin Sensitisation: Assays addressing the Adverse Outcome Pathway key event on covalent binding to proteins. OECD Guidelines for the Testing of Chemicals, Section 4. OECD Publishing Paris. doi:10.1787/9789264229709-en

OECD (2017a). Guidance Document on the Reporting of Defined Approaches to be Used Within Integrated Approaches to Testing and Assessment. OECD Series on Testing and Assessment, No. 255. OECD Publishing, Paris. doi:10.1787/9789264274822-en

OECD (2017b). Guidance Document on the Reporting of Defined Approaches and Individual Information Sources to be Used Within Integrated Approaches to Testing and Assessment (IATA) for Skin Sensitisation. OECD Series on Testing and Assessment, No. 256. OECD Publishing, Paris. doi:10.1787/9789264279285-en

OECD (2018a). Test No. 442E: In Vitro Skin Sensitisation Assays Addressing the Key Event on Activation of Dendritic Cells on the Adverse Outcome Pathway for Skin Sensitisation. OECD Guideline for the Testing of Chemicals, Section 4. OECD Publishing, Paris. doi:10.1787/9789264264359-en

OECD (2018b). Test No. 442D: In Vitro Skin Sensitisation: ARE-Nrf2 Luciferase Test Method. OECD Guideline for the Testing of Chemicals, Section 4. OECD Publishing, Paris. doi:10.1787/9789264229822-en

Piroird, C., Ovigne, J. M., Rousset, F. et al. (2015). The myeloid U937 skin sensitization test (U-SENS) addresses the activation of dendritic cell event in the adverse outcome pathway for skin sensitization. Toxicol In Vitro 29, 901-916. doi:10.1016/j. tiv.2015.03.009

Ramirez, T., Mehling, A., Kolle, S. N. et al. (2014). LuSens: A keratinocyte based ARE reporter gene assay for use in inte- grated testing strategies for skin sensitization hazard identification. Toxicol In Vitro 28, 1482-1497. doi:10.1016/j. tiv.2014.08.002

Rudback, J., Hagvall, L., Borje, A. et al. (2014). Characterization of skin sensitizers from autoxidized citronellol - Impact of the terpene structure on the autoxidation process. Contact Dermatitis 70, 329-339. doi:10.1111/cod.12234

Schellenberger, M., Bock, U., Hennen, J. et al. (2019). A coculture system composed of THP-1 cells and 3D reconstructed human epidermis to assess activation of dendritic cells by sensitizing chemicals after topical exposure. Toxicol In Vitro 57, 62-66. doi:10.1016/j.tiv.2019.02.002

Takahashi, T., Kimura, Y., Saito, R. et al. (2011). An in vitro test to screen skin sensitizers using a stable THP-1-derived IL-8 reporter cell line, THP-G8. Toxicol Sci 124, 359-369. doi:10.1093/toxsci/kfr237

Takenouchi, O., Miyazawa, M., Saito, et al. (2013). Predictive performance of the human Cell Line Activation Test (h-CLAT) for lipophilic chemicals with high octanol-water partition coefficients. Toxicol Sci 38, 599-609. doi 10.2131/jts.38.599

UN (2017). Globally Harmonized System of Classification and Labelling of Chemicals (GSH). Seventh revised edition. UN, New York. doi:10.18356/e9e7b6dc-en

Urbisch, D., Mehling, A., Guth, K. et al. (2015). Assessing skin sensitization hazard in mice and men using non-animal test methods. Regul Toxicol Pharmacol 71, 337-351. doi:10.1016/j.yrtph.2014.12.008

Wang, Y., Shibuya, K., Yamashita, Y. et al. (2008). LFA-1 decreases the antigen dose for $\mathrm{T}$ cell activation in vivo. Int Immunol 20, 1119-1127. doi:10.1093/intimm/dxn070

Wong, C. L., Ghassabian, S., Smith, M. T. et al. (2015). In vitro methods for hazard assessment of industrial chemicals - Opportunities and challenges. Front Pharmacol 6, 94. doi:10.3389/fphar.2015.00094

\section{Conflict of interest}

The authors have no conflicts of interest.

\section{Acknowledgments}

The authors thank Natalie Lobes for excellent technical assistance. This study was partly supported by the Federal Office of Public Health, Switzerland (11.004203) and by the Program for Women Professors of the Federal Ministry of Education and Research (Germany). 